\title{
THE EQUALITY OF 3-MANIFOLD INVARIANTS
}

\author{
John W. Barrett and Bruce W. Westbury
}

31 March 1995

\begin{abstract}
The invariants of 3-manifolds defined by Kuperberg for involutory Hopf algebras and those defined by the authors for spherical Hopf algebras are the same for Hopf algebras on which they are both defined.
\end{abstract}

\section{INTRODUCTION}

The purpose of this paper is to compare two previously defined invariants of 3-manifolds.

Let $A$ be a finite-dimensional Hopf algebra over a field $\mathbb{F}$ with antipode $S$. Then if $S^{2}=1$ the Hopf algebra is said to be involutory. Let $A$ be involutory and the dimension of $A$ in the field $\mathbb{F}$ be not zero. Then it follows from [Larson and Radford 1987] that $A$ is semisimple and cosemisimple.

For each such Hopf algebra $A$, Kuperberg [1990] has defined an invariant of closed oriented 3-manifolds. For a manifold $M$, this invariant is denoted $K(M)$.

The present authors defined an invariant of closed oriented 3-manifolds for each such Hopf algebra $A$ over an algebraically closed field [Barrett and Westbury 1993; proposition 6.8$]^{1}$. This invariant is denoted $Z(M)$ for a manifold $M$ and is called a state sum invariant.

The result of this paper is

Theorem. Let $A$ be a finite dimensional involutory Hopf algebra over an algebraically closed field, with $\operatorname{dim} A \neq 0$. Then

$$
K(M)=Z(M) \operatorname{dim} A .
$$

for all $M$.

This result implies the following relationship between the scope of the two invariants. The state sum invariants of Barrett and Westbury [1993] are defined for the more general notion of a finite semisimple spherical category of non-zero dimension. This generalises the notion of the category of representations of a semisimple Hopf algebra. As we showed in that paper, examples can be constructed from Hopf algebras which are not themselves semisimple, such as the quantised universal enveloping algebras. For these non-semisimple Hopf algebras it is apparently

\footnotetext{
${ }^{1}$ The hypothesis there that the field has characteristic zero can be replaced by the hypothesis that the algebra has non-zero dimension.
} 
necessary to use the category theory, as a quotient category has to be taken. $\mathrm{Ku}$ perberg's invariants are examples of the state sum invariants for which the category theory is not required.

Kaplansky's conjecture is that a finite-dimensional semisimple Hopf algebra is involutory. This has been proved for characteristic zero [Larson and Radford 1987]. If Kaplansky's conjecture is correct, then the state sum invariants which are defined directly from Hopf algebras without the category theory are all examples of Kuperberg's invariants if a particular element of the Hopf algebra, the spherical element, is taken to be 1 . There are further examples with other choices of spherical element.

\section{THE INVARIANTS}

The definitions of the two invariants are given here, followed by the proof of the theorem stated in the introduction. The definitions rely on various arbitrary choices; the proof that the invariants do not depend on these choices is not repeated here, but the reader may find these in the original references.

Before starting with the definitions, there are some preliminaries on Hopf algebras. Let $A$ be a finite dimensional involutory Hopf algebra of non-zero dimension over the algebraically closed field $\mathbb{F}$. If $a$ is a module over $A$, the dimension of $a$ is likewise the vector space dimension of $a$ regarded as an element of the field. The algebra $A$ can also be regarded as a left $A$-module, and $\operatorname{dim} A$ is unambiguous, the dimension of this module and the algebra $A$ being the same. The matrix trace for module $a$ is denoted $\operatorname{tr}_{a}$. Thus

$$
\operatorname{dim} a=\operatorname{tr}_{a}(1)
$$

The semisimplicity of $A$ has a number of consequences which follow. Let $I$ be complete set of inequivalent irreducible left $A$-modules with non-zero dimension. Thus

$$
\operatorname{dim} A=\sum_{a \in I}(\operatorname{dim} a)^{2} \neq 0 \in \mathbb{F}
$$

Also, if $a \in I$ and $b$ is any left $A$-module, then the pairing

$$
\begin{gathered}
\operatorname{Hom}_{A}(a, b) \times \operatorname{Hom}_{A}(b, a) \rightarrow \mathbb{F} \\
(\alpha, \beta) \mapsto \operatorname{tr}_{a} \beta \alpha
\end{gathered}
$$

is non-degenerate. In this paper, the composition of map $\alpha$ with $\beta$ is written $\beta \alpha$. This is the opposite convention to that of Barrett and Westbury [1993].

The algebra $A$ possesses a unique left integral $i \in A$ such that the counit $\epsilon$ gives $\epsilon(i)=1$ [Larson and Sweedler 1969]. For any left $A$-modules $a$ and $b, \operatorname{Hom}_{\mathbb{F}}(a, b)$ is also a left $A$-module, an element $x \in A$ acting by

$$
x: \phi \mapsto \sum \Delta_{(1)}(x) \phi S\left(\Delta_{(2)}(x)\right)
$$

where the coproduct is written 
The left integral $i$ has the property that $i^{2}=\epsilon(i) i$ in any Hopf algebra. In the semisimple case this gives $i^{2}=i$, and $i$ acts by projection in an $A$-module. For a non-semisimple Hopf algebra this would give $i^{2}=0$ and the following considerations would not apply. The subspace $\operatorname{Hom}_{A}(a, b) \subset \operatorname{Hom}_{\mathbb{F}}(a, b)$ is exactly the image of the projection given by the left action of the integral $i$.

The Hopf algebra $A$ has a unique left cointegral $c \in A^{*}$ with $c(1)=1$. This is given by

$$
c(x)=\frac{1}{\operatorname{dim} A} \operatorname{tr}_{A}(x)=\frac{1}{\operatorname{dim} A} \sum_{a \in I} \operatorname{dim} a \operatorname{tr}_{a}(x)
$$

[Larson and Radford 1987], where the first trace is over the left regular representation of $A$.

\section{The state sum invariant.}

The definition of the state sum invariant given here is the definition of Barrett and Westbury [1993] specialised to the case of involutory Hopf algebras with the spherical element equal to 1.

Let $M$ be an oriented closed 3-manifold, and fix a triangulation of $M$.

It is necessary to pick a simplicial structure for the triangulated manifold $M$. This means that a total ordering is chosen for the vertices of each simplex such that the orderings of faces are compatible. A suitable such structure can be obtained by totally ordering all the vertices of $M$.

Let $E$ be the set of edges (1-simplexes) of $M$ and $v$ the number of vertices of $M$. A labelling of $M$ is a map $l: E \rightarrow I$. For each labelled simplicial manifold $(M, l)$, a number $Z(M, l) \in \mathbb{F}$ is defined, and the invariant is determined from this data by a sum

$$
Z(M)=(\operatorname{dim} A)^{-v} \sum_{l: E \rightarrow I} Z(M, l) \prod_{e \in E} \operatorname{dim}(l(e))
$$

over the set of all labellings.

It remains to define $Z(M, l)$. For each triangle in $M$, there are three elements $e_{12}, e_{02}, e_{01}$ of $I$ assigned to the edges $12,02,01$. This determines a vector space, $\operatorname{Hom}_{A}\left(e_{02}, e_{12} \otimes e_{01}\right)$. The vector space $V(M, l)$ is defined to be the tensor product of each of these spaces over the set of all triangles in $M$.

For each tetrahedron in $M$, denote the labelling of the edge $(i j)$ by $e_{i j}$, for $i<$ $j=0, \ldots 3$. The ordering of the vertices determines an orientation of the simplex which either agrees (positive) with that of the manifold or does not (negative). These two cases are considered separately.

Consider first the case where the simplex is positive. There are maps

$$
\begin{aligned}
\operatorname{Hom}_{A}\left(e_{03}, e_{23} \otimes e_{02}\right) \otimes \operatorname{Hom}_{A}\left(e_{02}, e_{12} \otimes e_{01}\right) & \\
& \rightarrow \operatorname{Hom}_{A}\left(e_{03}, e_{23} \otimes e_{12} \otimes e_{01}\right)
\end{aligned}
$$

and

(9) $\operatorname{Hom}_{A}\left(e_{13}, e_{23} \otimes e_{12}\right) \otimes \operatorname{Hom}_{A}\left(e_{03}, e_{13} \otimes e_{01}\right)$ 
If $A$ is semisimple, then these maps give isomorphisms

$$
\begin{aligned}
\bigoplus_{e_{02}} \operatorname{Hom}_{A}\left(e_{03}, e_{23} \otimes e_{02}\right) \otimes \operatorname{Hom}_{A}\left(e_{02}, e_{12} \otimes e_{01}\right) \\
\rightarrow \operatorname{Hom}_{A}\left(e_{03}, e_{23} \otimes e_{12} \otimes e_{01}\right)
\end{aligned}
$$

and

$$
\begin{aligned}
\bigoplus_{e_{13}} \operatorname{Hom}_{A}\left(e_{13}, e_{23} \otimes e_{12}\right) \otimes \operatorname{Hom}_{A}\left(e_{03}, e_{13} \otimes e_{01}\right) \\
\rightarrow \operatorname{Hom}_{A}\left(e_{03}, e_{23} \otimes e_{12} \otimes e_{01}\right) .
\end{aligned}
$$

Comparing these two decompositions gives a map

$$
\begin{aligned}
\operatorname{Hom}_{A}\left(e_{03}, e_{23} \otimes e_{02}\right) \otimes & \operatorname{Hom}_{A}\left(e_{02}, e_{12} \otimes e_{01}\right) \\
& \rightarrow \operatorname{Hom}_{A}\left(e_{13}, e_{23} \otimes e_{12}\right) \otimes \operatorname{Hom}_{A}\left(e_{03}, e_{13} \otimes e_{01}\right) .
\end{aligned}
$$

Our definition, which follows, will give a map which is proportional to this one, in fact by dividing by $\operatorname{dim}\left(e_{13}\right)$. This definition is the one used in Barrett and Westbury [1993]. The constant of proportionality is given there by lemma 5.4.

The definition uses the fact that the trace gives a non-degenerate bilinear form, but without necessarily assuming directly that $A$ is semi-simple.

Note that if

$$
\begin{aligned}
& \beta \otimes \delta \in \operatorname{Hom}_{A}\left(e_{03}, e_{23} \otimes e_{02}\right) \otimes \operatorname{Hom}_{A}\left(e_{02}, e_{12} \otimes e_{01}\right) \\
& \alpha \otimes \gamma \in \operatorname{Hom}_{A}\left(e_{23} \otimes e_{12}, e_{13}\right) \otimes \operatorname{Hom}_{A}\left(e_{13} \otimes e_{01}, e_{03}\right)
\end{aligned}
$$

then

$$
\begin{aligned}
& \gamma(\alpha \otimes 1): e_{23} \otimes e_{12} \otimes e_{01} \rightarrow e_{03} \\
& (1 \otimes \delta) \beta: e_{03} \rightarrow e_{23} \otimes e_{12} \otimes e_{01} .
\end{aligned}
$$

Therefore a map (12) is uniquely determined by the condition that $\beta \otimes \delta$ maps to the unique element whose pairing with $\alpha \otimes \gamma$ is

$$
\operatorname{tr}(\gamma(\alpha \otimes 1)(1 \otimes \delta) \beta),
$$

using the non-degenerate bilinear form.

If the simplex is negative, a map

$$
\begin{aligned}
\operatorname{Hom}_{A}\left(e_{13}, e_{23} \otimes e_{12}\right) \otimes \operatorname{Hom}_{A} & \left(e_{03}, e_{13} \otimes e_{01}\right) \\
\rightarrow & \operatorname{Hom}_{A}\left(e_{03}, e_{23} \otimes e_{02}\right) \otimes \operatorname{Hom}_{A}\left(e_{02}, e_{12} \otimes e_{01}\right)
\end{aligned}
$$

is defined by mapping $\alpha \otimes \gamma$ to the unique element which has trace

$$
\operatorname{tr}(\beta(1 \otimes \delta)(\alpha \otimes 1) \gamma)
$$

with $\beta \in \operatorname{Hom}_{A}\left(e_{23} \otimes e_{02}, e_{03}\right)$ tensored with $\delta \in \operatorname{Hom}_{A}\left(e_{12} \otimes e_{01}, e_{02}\right)$, for all $\beta, \delta$.

The tensor product of the maps (13) and (14) over the set of all tetrahedra in the manifold is a linear map $V(M, l) \rightarrow V^{\pi}(M, l)$, where $V^{\pi}(M, l)$ is defined in the same way as $V(M, l)$ but with the factors permuted by some permutation $\pi$. There is a unique standard linear map $V^{\pi}(M, l) \rightarrow V(M, l)$ given by iterating the standard twist $P: x \otimes y \mapsto y \otimes x$. This defines an element of End $V(M, l)$, and 


\section{The Kuperberg invariant.}

Kuperberg's invariant [Kuperberg 1990] is defined by assigning a number to a Heegaard diagram for an oriented closed 3-manifold. For the purposes of this paper it is sufficient to give a definition for the particular Heegaard diagrams determined by a triangulation.

The triangulation of $M$ determines a Heegaard splitting for $M$ by a regular neighbourhood of the 1-skeleton. This can be done in a standard way by taking a subcomplex of the second barycentric subdivision of $M$. The triangulation determines standard circles on the Heegaard surface; there is one upper circle for each edge of the triangulation and one lower circle for each face. The lower circles are defined to be the intersection of the face with the Heegaard surface and the upper circles are defined to be the intersection of the dual face in the dual skeleton with the Heegaard surface. The Heegaard surface together with the upper and lower circles determines the manifold up to isomorphism and is called a Heegaard diagram for the manifold.

An arbitrary orientation and a distinguished point is chosen for each circle. Starting at the distinguished point and travelling in the direction of the orientation, this gives an ordering of the crossing points between upper and lower circles which occur around a given circle. The set of upper circles and the set of lower circles are also regarded as ordered sets. This endows the set of all crossing points with two distinct total orderings; one is obtained by traversing all the upper circles in order, the other by traversing all the lower circles in order.

The Heegaard surface is oriented as the boundary of the thickened 1-skeleton. This determines a sign for each crossing point as positive if there is a positively oriented triangle (012) embedded in the Heegaard surface with vertex 0 at the crossing point, 1 along the upper circle in the direction of its orientation and 2 along the lower circle. Otherwise it is negative.

Let $l, u$ be the number of lower and upper circles and $n$ the number of crossing points. Each lower circle has exactly three crossing points. The element

$$
k=(\Delta \otimes 1) \Delta(i) \in A \otimes A \otimes A
$$

is associated with each lower circle, with one factor associated to each crossing point. For each crossing point, define the linear map $\phi: A \rightarrow A$ to be the identity if the crossing point is positive and the antipode $S$ if the crossing point is negative. The linear functional

$$
T:\left(x_{1}, x_{2}, \ldots\right) \mapsto c\left(\phi\left(x_{1}\right) \phi\left(x_{2}\right) \ldots\right)
$$

on $A \otimes A \otimes \ldots$ is associated to each upper circle, with one factor associated to each crossing point, using the $\phi$ appropriate for each crossing point.

A number $\lambda \in \mathbb{F}$ is determined by applying the permutation which takes the total order determined by the lower circles to the total order determined by the upper circles to the element

$$
\otimes^{l} k \in \otimes^{n} A
$$

followed by the linear functional $\otimes^{n} A \rightarrow \mathbb{F}$ obtained by tensoring the $T$ for each upper circle. As before, the permuations act in the linear space $\otimes^{n} A$ by iterates of the standard twist map $P$.

The Kuperberg invariant $K(M)$ is defined to be

$$
K(M)=\lambda(\operatorname{dim} A)^{u-v+1} .
$$




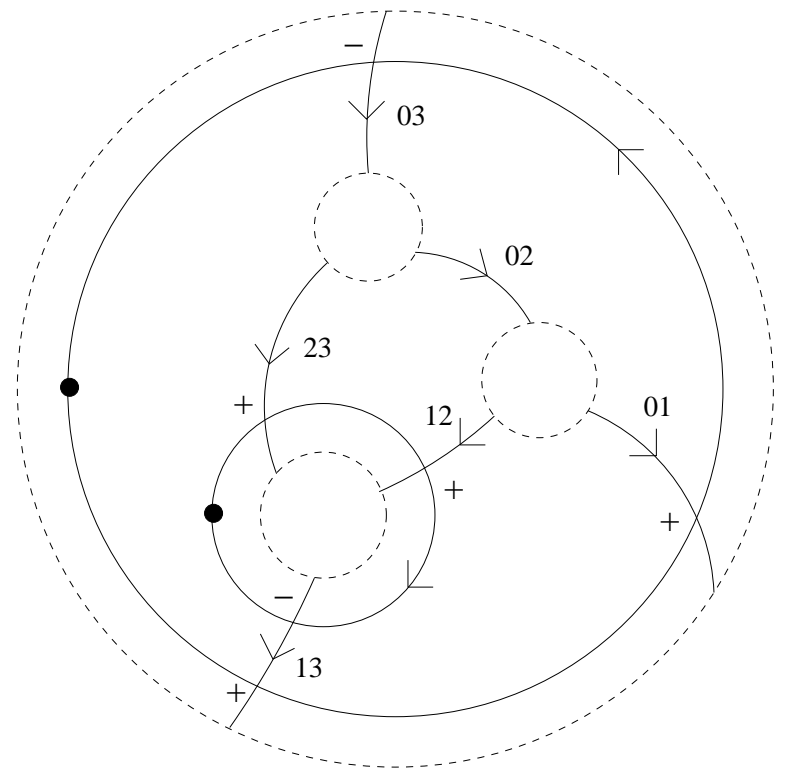

FIGURE 1

\section{The proof of the theorem.}

Since the edges and upper circles correspond, a labelling assigns a module in $I$ to each upper circle. In the definition of $\lambda$ in the invariant $K(M)$, replace each $T$ for each upper circle with the functional

$$
\left(x_{1}, x_{2}, \ldots\right) \mapsto \operatorname{tr}_{a}\left(\phi\left(x_{1}\right) \phi\left(x_{2}\right) \ldots\right)
$$

$a$ being the module assigned to the given upper circle. Denote this element of $\mathbb{F}$ by $\lambda(M, l)$. Then, using $(6), K(M)$ is given by the formula

$$
K(M)=(\operatorname{dim} A)^{1-v} \sum_{l: E \rightarrow I} \lambda(M, l) \prod_{e \in E} \operatorname{dim}(l(e))
$$

Comparing this with the definition (7) of the state sum invariant, to prove the theorem it remains to show that $Z(M, l)=\lambda(M, l)$ for each labelling $l$.

The Heegaard surface can be split into the union of its intersection with each tetrahedron in the manifold. The lower circles are on the boundary of each of these pieces; to simplify the proof they are slid sideways a small amount so that they lie in a single tetrahedron. The rule for doing this is that the lower circle lies in the tetrahedron for which the face which corresponds to it is positively signed in the boundary of the tetrahedron. This is according to the convention

$$
\partial+(0123)=+(123)-(023)+(013)-(012)
$$

and with the opposite signs for -(0123). Also, the orientations of the circles are chosen with respect to the orderings of the vertices of $M$. The ordering of the vertices gives an orientation for each edge of the triangulation, and using the orientation of the manifold, a rule can be chosen to orient the upper circles in a consistent way. These circles can be chosen to circulate the edges in a clockwise or anti-clockwise sense throughout the manifold. This choice is fixed by the orientations indicated in 
tetrahedron $+(0123)$ is shown. This is a disk with three punctures. The dotted line is the boundary, while the solid line segments are the upper circles, labelled with the corresponding edge, and the solid line circles are lower circles. The signs,+on the diagram indicate the signs of the crossing points, which are determined by the orientations. A point is marked on each lower circle as the distinguished point. Figure 2 is the same diagram for a negatively oriented tetrahedron.

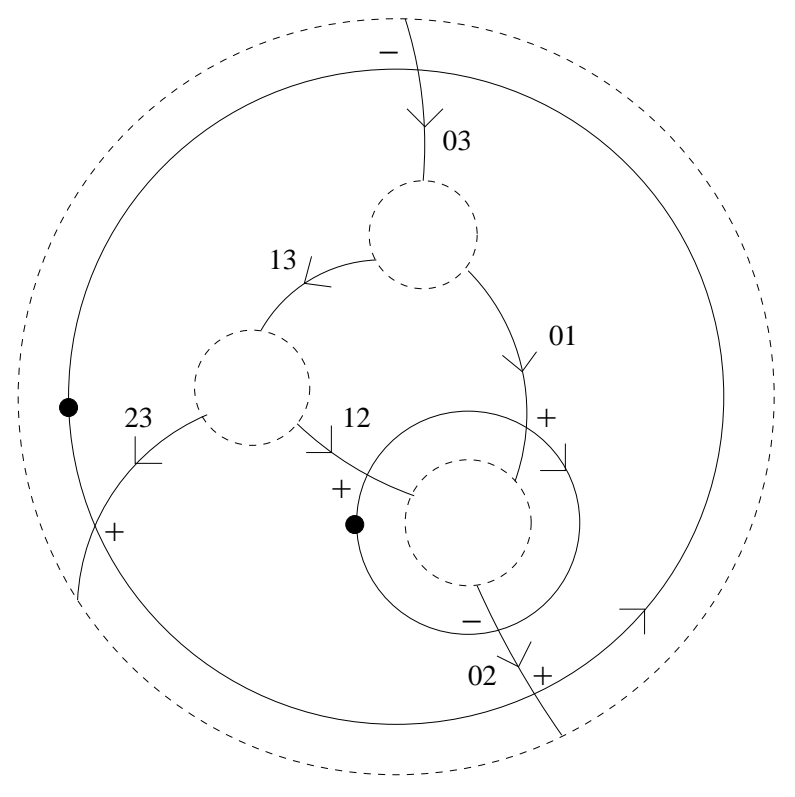

FIGURE 2

Each lower circle in Figures 1 and 2 has been chosen so that the signs of the crossing points are $(+,+,-)$, as in Figure 3 . Figure 3 shows part of a Heegaard surface, with the upper circles labelled by $a, b$ and $c$. The number $\lambda(M, l)$ is calculated by

$$
t r_{a \otimes b \otimes c}(((1 \otimes 1 \otimes S)(k)) \eta),
$$

where $k$ is defined in (15) and

$$
\eta \in \operatorname{End}_{\mathbb{F}}(a \otimes b \otimes c)
$$

is the element determined in the definition of $\lambda(M, l)$ for the rest of the Heegaard diagram, outside Figure 3. Thus $\lambda(M, l)$ can be considered to be the value of a certain linear functional acting on the element $\eta$.

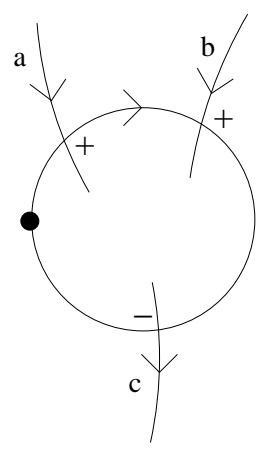


For fixed elements $\alpha \in \operatorname{Hom}_{\mathbb{F}}(a \otimes b, c), \beta \in \operatorname{Hom}_{\mathbb{F}}(c, a \otimes b)$, there is a rank one endomorphism of $\operatorname{Hom}_{\mathbb{F}}(c, a \otimes b)$ defined by

$$
x \mapsto\left(\operatorname{tr}_{c}(\alpha x)\right) \beta .
$$

The matrix trace of this endomorphism is $\operatorname{tr}_{c}(\alpha \beta)$, and the set of all such endomorphisms for all $\alpha$ and $\beta$ spans this linear space of endomorphisms, using the hypothesis that $A$ is semisimple, and (3). There is an isomorphism

$$
\operatorname{End}_{\mathbb{F}}\left(\operatorname{Hom}_{\mathbb{F}}(c, a \otimes b)\right) \rightarrow \operatorname{End}_{\mathbb{F}}(a \otimes b \otimes c)
$$

determined by

$$
\beta \operatorname{tr}_{c}(\alpha \cdot) \mapsto P(\alpha \otimes \beta)
$$

with $P$ the usual twist map $c \otimes(a \otimes b) \rightarrow(a \otimes b) \otimes c$. Using this isomorphism, the linear functional on $\operatorname{End}_{\mathbb{F}}\left(\operatorname{Hom}_{\mathbb{F}}(c, a \otimes b)\right)$ corresponding to (19) is given by

$$
\beta \operatorname{tr}_{c}(\alpha \cdot) \mapsto \operatorname{tr}_{c}\left(\alpha \Delta_{(1)}(i) \beta S\left(\Delta_{(2)}(i)\right)\right) .
$$

This is the trace on the vector space $\operatorname{Hom}_{\mathbb{F}}(c, a \otimes b)$ with the map

$$
\beta \mapsto \Delta_{(1)}(i) \beta S\left(\Delta_{(2)}(i)\right) .
$$

As explained following (5), this map is a projection on $\operatorname{Hom}_{A}(c, a \otimes b)$.

This method can be repeated for the whole of Figure 1, using the isomorphism of

$$
\operatorname{End}_{\mathbb{F}}\left(e_{01} \otimes e_{02} \otimes e_{03} \otimes e_{12} \otimes e_{13} \otimes e_{23}\right)
$$

with

$$
\begin{aligned}
\operatorname{Hom}_{\mathbb{F}}\left(\operatorname{Hom}_{\mathbb{F}}\left(e_{03}, e_{23} \otimes e_{02}\right) \otimes\right. & \operatorname{Hom}_{\mathbb{F}}\left(e_{02}, e_{12} \otimes e_{01}\right), \\
& \left.\operatorname{Hom}_{\mathbb{F}}\left(e_{13}, e_{23} \otimes e_{12}\right) \otimes \operatorname{Hom}_{\mathbb{F}}\left(e_{03}, e_{13} \otimes e_{01}\right)\right) .
\end{aligned}
$$

Since each lower circle determines a projection on $\operatorname{Hom}_{A}(a, b \otimes c)$, each space $\operatorname{Hom}_{\mathbb{F}}(a, b \otimes c)$ can be replaced by $\operatorname{Hom}_{A}(a, b \otimes c)$. A short calculation shows that the part of the Heegaard diagram in Figure 1 determines the same linear map as that associated to the positive tetrahedron in the state sum invariant. A similar calculation can be carried out for the negative tetrahedra in the manifold. This shows that $\lambda(M, l)=Z(M, l)$.

\section{REFERENCES}

J.W. Barrett and B.W. Westbury, Invariants of piecewise-linear 3-manifolds (1993).

G. Kuperberg, Involutory Hopf algebras and 3-manifold invariants, Int. J. Math. 2 (1990), 41-66. R.G. Larson and D.E. Radford, Semisimple cosemisimple Hopf algebras, Amer. J. Math. 109 (1987), 187-195.

R.G. Larson and M.E. Sweedler, An associative orthogonal bilinear form for Hopf algebras, Amer.

J. Math. 91 (1969), 75-94.

Department of Mathematics, University of Nottingham, University Park, NotTINGHAM, NG7 2RD

E-mail address: jwb@maths.nott.ac.uk

Department of Mathematics, University of Nottingham, University Park, NotTINGHAM, NG7 2RD 